2. Borovikov S. N., Kryukov I. A., Ivanov I. E. Unstructured triangular mesh generation on curved faces based on Delauney triangulation. Matem. Mod., 2005, vol. 17, no. 8, pp. 31-45 (in Russian).

3. Shewchuk J. R. What is a Good Linear Element? Interpolation, Conditioning and Quality Measures. Preprint / Department of Electrical Engineering and Computer Sciences University of California at Berkeley, Berkeley, 2002, CA 94720.

4. Gelbaum B. R., Olmsted J. M. H. Counterexamples in analysis. San Francisco, London, Amsterdam, Holden-Day, 1964.

5. Klyachin V. A., Shirokii A. A. The Delaunay triangulation for multidimensional surfaces and its approximative properties. Russian Math. [Izvestiya VUZ. Matematika], 2012, vol. 56, no. 1, pp. 27-34. DOI: $10.3103 /$ S1066369X12010045.

6. Klyachin V. A., Pabat E. A. $C^{1}$-approximation of the level surfaces of functions defined on irregular grids. Sib. Zh. Ind. Mat., 2010, vol. 13, no. 2, pp. 69-78 (in Russian).

7. Skvortsov A. V., Mirza N. S. Algoritmy postroeniya $i$ analiza triangulacii [Constructing and Analisys of Triangulation Algorithms] Tomsk, Tomsk Univ. Press, 2006 (in Russian).

8. Klyachin V. A. On a multidimensional analogue of the Schwarz example. Izv. Math., 2012, vol. 76 , no. 4 , pp. 681-687. DOI: 10.1070/IM2012 v076n04ABEH002601.

9. Ushakova O. V. Nondegeneracy conditions for three-dimensional cells and a formula for the cell's volume. Comput. Math. Math. Phys., 2001, vol. 41, no. 6 , pp. 832-845.

10. Berger M. Geometry Translated from the French by $M$. Cole and S. Levy. Universitext. Berlin, Springer-Verlag, 1987. xiv+428 p.

\title{
ASYMPTOTIC RATIO OF HARMONIC MEASURES OF SLIT SIDES
}

\section{V. Prokhorov, D. V. Ukrainskii}

Saratov State University, 83, Astrakhanskaya st., 410012, Saratov, Russia, ProkhorovDV @ info.sgu.ru, D.V.Ukrainskiy@gmail.com

The article is devoted to the geometry of solutions to the chordal Löwner equation which is based on the comparison of singular solutions and harmonic measures for the sides of a slit in the upper half-plane generated by a driving term. An asymptotic ratio for harmonic measures of slit sides is found for a slit which is tangential to a straight line under a given angle, and for a slit with high order tangency to a circular arc tangential to the real axis.

Key words: Löwner equation, singular solution, harmonic measure, half-plane capacity.

\section{INTRODUCTION}

The famous Löwner differential equation have been introduced in 1923 [1] and was aimed to give a parametric representation of slit domains. In this article we describe an asymptotic behavior of singular solutions and harmonic measures for the sides of a slit in domains generated by a driving term of the Löwner equation.

The chordal version of the Löwner equation deals with the upper half-plane $\mathbb{H}=\{z: \operatorname{Im} z>0\}$, $\mathbb{R}=\partial \mathbb{H}$, and functions $f(z, t)$ normalized near infinity by

$$
f(z, t)=z+\frac{2 t}{z}+O\left(\frac{1}{z^{2}}\right)
$$

which solve the chordal Löwner differential equation

$$
\frac{d f(z, t)}{d t}=\frac{2}{f(z, t)-\lambda(t)}, \quad f(z, 0) \equiv z, \quad t \geq 0,
$$

and map subdomains of $\mathbb{H}$ onto $\mathbb{H}$. Here $\lambda(t)$ is a real-valued continuous driving term.

Let $\gamma_{t}:=\gamma[0, t]=\{\gamma(x): 0 \leq x \leq t\}$ be a simple continuous curve in $\mathbb{H} \cup\{0\}$ with endpoints $\gamma(0)=0$ and $\gamma(t), 0 \leq t \leq T$. Then there is a unique map $f(z, t): \mathbb{H} \backslash \gamma_{t} \rightarrow \mathbb{H}$ satisfying the chordal Löwner equation (1) with $\lambda(t)$ uniquely determined by $\gamma[0, t]$. The function $f(z, t)$ can be extended continuously to $\mathbb{R} \cup \gamma(t)$, and $f(\gamma(t), t)=\lambda(t)$. The value $t$ is called the half-plane capacity of the curve $\gamma_{t}, t=h \operatorname{cap}\left(\gamma_{t}\right)$, see, e.g. [2].

We say that $\gamma_{t} \in C^{n}, n \in \mathbb{N}$, on $[0, S]$ if, for the arc-length parameter $s$ of $\gamma_{t}, \gamma(t(s))$ has a continuous derivative $\gamma^{(n)}$ in $s$ on $[0, S], t(S)=T$. All the derivatives $\gamma^{(k)}, 1 \leq k \leq n$, at $s=0$ are understood as one-side derivatives. A curve $\gamma_{t} \in C^{n}, \gamma(0)=0$, is said to have at least $n$-order tangency with a ray $I_{\theta}=\left\{e^{i \theta} s: s \geq 0\right\}, \theta \in \mathbb{R}$, at $s=0$ if

$$
\gamma(t(s))=e^{i \theta} s+o\left(s^{n}\right), \quad s \rightarrow+0 .
$$


Two curves $\gamma[0, s] \in C^{n}$ and $\Gamma[0, s] \in C^{n}$ are said to have at least $n$-order tangency at $s=0$ if derivatives $\gamma^{(k)}$ and $\Gamma^{(k)}$ in $s$ at $s=0$ coincide, $0 \leq k \leq n$.

The extended function $f(z, t)$ maps $\gamma_{t}$ onto a segment $I=I(t)=\left[f_{2}(0, t), f_{1}(0, t)\right]$ while $f(\mathbb{R})=\mathbb{R} \backslash I$. The function $f_{1}(0, t)$ is the maximal singular solution to the chordal Löwner equation $(1)$, and $f_{2}(0, t)$ is the minimal singular solution to (1). Both of these solutions correspond to the singular point $f(0,0)=0$ of equation (1).

The curve $\gamma_{t}$ has two sides $\gamma_{1 t}$ and $\gamma_{2 t}$ which define different prime ends at the same points, except for its tip. We say that $\gamma_{1 t}$ is the left side of $\gamma_{t}$ if going along the boundary of the domain $\mathbb{H} \backslash \gamma_{t}$ and moving along $\mathbb{R}$ from $(-\infty)$ to 0 , we first meet the side $\gamma_{1 t}$ and then $\gamma_{2 t}$. In this case, $\gamma_{2 t}$ is called the right side of $\gamma_{t}$. The two parts $\left[f_{2}(0, t), \lambda(t)\right]$ and $\left[\lambda(t), f_{1}(0, t)\right]$ of segment $I(t)$ are the images of the two sides $\gamma_{1 t}$ and $\gamma_{2 t}$ of $\gamma_{t}$ under $f(z, t)$, respectively.

The harmonic measures $\omega\left(f^{-1}(i, t) ; \gamma_{k t}, \mathbb{H} \backslash \gamma_{t}\right)$ of $\gamma_{k t}$ at $f^{-1}(i, t)$ with respect to $\mathbb{H} \backslash \gamma_{t}$ are defined by the functions $\omega_{k}$ which are harmonic on $\mathbb{H} \backslash \gamma_{t}$ and continuously extended on its closure except for the endpoints of $\gamma_{t},\left.\omega_{k}\right|_{\gamma_{k t}}=1,\left.\omega_{k}\right|_{\mathbb{R} \cup\left(\gamma_{t} \backslash \gamma_{k t}\right)}=0, k=1,2$, see, e.g., [3, $\left.\S 3.6\right]$. Denote

$$
m_{k}(t):=\omega\left(f^{-1}(i, t) ; \gamma_{k t}, \mathbb{H} \backslash \gamma_{t}\right), \quad k=1,2
$$

In Section 1, we prove the following theorem.

Theorem 1. Let $\gamma_{t} \in C^{4}, \gamma(0)=0, \operatorname{Im} \gamma(t)>0$ for $t>0$, have at least 4-order tangency at the origin to the straight line under the angle $\frac{\pi}{2}(1-\beta),-1<\beta<1$, to the real axis $\mathbb{R}$, and let $f(z, t)$ map $\mathbb{H} \backslash \gamma_{t}$ onto $\mathbb{H}$ and solve the chordal Löwner equation (1). Then

$$
\lim _{t \rightarrow+0} \frac{m_{1}(t)}{m_{2}(t)}=\frac{1+\beta}{1-\beta},
$$

where

$$
m_{k}(t):=\omega\left(f^{-1}(i, t) ; \gamma_{k t}, \mathbb{H} \backslash \gamma_{t}\right), \quad k=1,2,
$$

$\gamma_{1 t}$ is the left side of $\gamma_{t}$, and $\gamma_{2 t}$ is the right side of $\gamma_{t}$.

The most important argument in the proof is the comparison of asymptotic parametric representations of $\gamma_{t}$ in $t$ and $s$ at $s=0$. This approach can be compared with the result by Earle and Epstein [4].

In Section 2, we solve a similar problem for a curve $\gamma_{t}$ which has at least 6-order tangency with a circular arc in $\mathbb{H} \cup\{0\}$ tangential to $\mathbb{R}$ at the origin. Since a scaling time change $t \rightarrow \alpha^{2} t$ in the Löwner equation (1) is accompanied by changing $\lambda(t) \rightarrow \frac{1}{\alpha} \lambda\left(\alpha^{2} t\right)$ and $f(z, t) \rightarrow \frac{1}{\alpha} f\left(\alpha z, \alpha^{2} t\right), \alpha \in \mathbb{R}$, we can assume without loss of generality that the circular arc is of radius 1 , and the argument of its points is increasing when going from 0 . We prove the following theorem.

Theorem 2. Let $\gamma_{t} \in C^{6}, \gamma(0)=0, \operatorname{Im} \gamma(t)>0$ and $\operatorname{Re} \gamma(t)>0$ for $t>0$, have at least 6-order tangency at the origin to the circular arc of radius 1 centered at $i$, and let $f(z, t)$ map $\mathbb{H} \backslash \gamma_{t}$ onto $\mathbb{H}$ and solve the chordal Löwner equation (1). Then

$$
\lim _{t \rightarrow+0} \frac{M_{1}^{2}(t)}{M_{2}(t)}=2 \pi
$$

where

$$
M_{k}(t):=\omega\left(f^{-1}(i, t) ; \gamma_{k t}, \mathbb{H} \backslash \gamma_{t}\right), \quad k=1,2,
$$

$\gamma_{1 t}$ is the left side of $\gamma_{t}$, and $\gamma_{2 t}$ is the right side of $\gamma_{t}$.

\section{PROOF OF THEOREM 1}

Proof of Theorem 1. For $\beta=0$, Theorem 1 has been proved [5]. The cases $\beta>0$ and $\beta<0$ are symmetric to each other, and we will stop only on $\beta>0$.

The Löwner equation (1) can be integrated in quadratures in particular cases [6]. For example, if $\lambda(t)=c \sqrt{t}, c \geq 0$, then a solution $f_{c}(z, t)$ to equation (1) maps $\mathbb{H} \backslash \gamma_{t}$ onto $\mathbb{H}$ where $\gamma_{t}$ is parameterized as

$$
\gamma[0, t]=\{z=B \sqrt{x}: 0 \leq x \leq t\},
$$


with $B=B(c)=|B(c)| e^{i \theta(c)}$,

$$
|B(c)|=2\left(\frac{\sqrt{c^{2}+16}+c}{\sqrt{c^{2}+16}-c}\right)^{\frac{c}{2 \sqrt{c^{2}+16}}}, \quad \theta(c)=\frac{\pi}{2}\left(1-\frac{c}{\sqrt{c^{2}+16}}\right) .
$$

Suppose that a $C^{4}$-slit $\gamma_{t}$ satisfies the conditions of Theorem 1. Then there exists a driving function $\lambda(t) \in \operatorname{Lip}\left(\frac{1}{2}\right)$ such that a solution $w=f(z, t)$ to equation (1) maps $\mathbb{H} \backslash \gamma_{t}$ onto $\mathbb{H}$. For the arc-length parameter $s, \gamma(t(s))$ is represented as

$$
\gamma(t(s))=e^{i \theta} s+o\left(s^{4}\right), \quad s \rightarrow+0 .
$$

Denote

$$
I_{\theta}(t)=\left\{x e^{i \theta}: 0 \leq x \leq t\right\}, \quad 0<\theta<\frac{\pi}{2}, \quad t>0 .
$$

There is $c>0$ such that

$$
\beta=\frac{c}{\sqrt{c^{2}+16}}
$$

for which $\theta=\theta(c)=\frac{\pi}{2}(1-\beta)$. Then $f_{c}(z, \tau)$ maps $\mathbb{H} \backslash I_{\theta(c)}(|B(c)| \sqrt{\tau})$ onto $\mathbb{H}$. The length $\sigma(\tau)$ of $I_{\theta(c)}(|B(c)| \sqrt{\tau})$ and the half-plane capacity $\tau$ of $I_{\theta(c)}(|B(c)| \sqrt{\tau})$ are related by

$$
\sigma(\tau)=|B(c)| \sqrt{\tau}, \quad \tau>0 .
$$

Let $s$ denote the length of $\gamma_{t(s)}$ and let $\sigma$ denote the length of projection of $\gamma_{t(s)}$ onto $I_{\theta(c)}(T)$. There is a $C^{4}$-dependence $s=s(\sigma)$,

$$
s(0)=0, \quad s^{\prime}(0)=1, \quad s^{(k)}(0)=0, \quad k=2,3,4 .
$$

Therefore,

$$
s=\sigma+o\left(\sigma^{4}\right), \quad \sigma \rightarrow+0 .
$$
on $I_{\theta}$,

Asymptotic expansion (2) implies an asymptotic behavior of a distance between $\gamma_{t}$ and its projection

$$
\operatorname{dist}\left(\gamma_{t(s)}, I_{\theta(c)}(\sigma(s))\right)=o\left(\sigma^{4}(s)\right), \quad s \rightarrow+0 .
$$

Lind, Marshall and Rohde [2] studied the closeness of half-plane capacities for two curves which are close together. According to Lemma 4.10 [2], we have that

$$
t(s)-\tau(\sigma(s))=o\left(s^{2}\right), \quad s \rightarrow+0,
$$

where $\sigma(\tau)$ is given by (4). Hence, due to (4) and (5),

$$
t(s(\sigma))=\tau(\sigma)+o\left(s^{2}(\sigma)\right)=\tau(\sigma)+o\left(\sigma^{2}\right)=|B(c)|^{-2} s^{2}+o\left(s^{2}\right), \quad s \rightarrow+0 .
$$

Take into account (2) and rewrite the last relation in the form

$$
\gamma(t)=|B(c)| \sqrt{t}+\alpha(t) \sqrt{t}, \quad \lim _{t \rightarrow+0} \alpha(t)=0 .
$$

Choose an arbitrary sequence $\left\{x_{n}\right\}$ of positive numbers $x_{n}, x_{n} \rightarrow \infty$ as $n \rightarrow \infty$, and denote

$$
z=g_{n}(w, t):=\sqrt{x_{n}} f^{-1}\left(\frac{w}{\sqrt{x_{n}}}, \frac{t}{x_{n}}\right), \quad n=1,2, \ldots .
$$

The function $z=f^{-1}(w, t)$ maps $\mathbb{H}$ onto $\mathbb{H} \backslash \gamma[0, t], f^{-1}(\lambda(t), t)=\gamma(t)$. So the functions $g_{n}(w, t)$ map $\mathbb{H}$ onto $\mathbb{H} \backslash \gamma^{(n)}[0, t]$ where

$$
\begin{gathered}
\gamma^{(n)}(t)=\sqrt{x_{n}} \gamma\left(\frac{t}{x_{n}}\right)=e^{i \theta(c)}|B(c)| \sqrt{t}+\alpha\left(\frac{t}{x_{n}}\right) \sqrt{t}, \\
g_{n}\left(\sqrt{x_{n}} \lambda\left(\frac{t}{x_{n}}\right), t\right)=\gamma^{(n)}(t), \quad 0<t \leq T .
\end{gathered}
$$


We see that

$$
\gamma^{(n)}(t)-e^{i \theta(c)}|B(c)| \sqrt{t}=\alpha\left(\frac{t}{x_{n}}\right) \sqrt{t} \rightarrow 0, \quad n \rightarrow \infty,
$$

and the convergence is uniform with respect to $t \in[0, T]$.

The Radó theorem [7], see also [8, p. 60], states that a sequence $\left\{h_{n}\right\}$ of conformal mappings $h_{n}$ from the unit disk $\mathbb{D}$ onto simply connected domains $D_{n}$ bounded by Jordan curves $\partial D_{n}, 0 \in D_{n}, h_{n}(0)=0$, $h_{n}^{\prime}(0)>0$, converges uniformly on the closure of $\mathbb{D}$ to $h: \mathbb{D} \rightarrow D, \partial D$ is bounded by a Jordan curve, if and only if $D_{n}$ converges to the kernel $D$ and, for every $\epsilon>0$, there exists $N>0$ such that, for all $n>N$, there is a one-to-one correspondence $z_{n}: \partial D_{n} \rightarrow \partial D,\left|z_{n}(\zeta)-\zeta\right|<\epsilon, \zeta \in \partial D_{n}$. Markushevich [9] generalized the Radó theorem to domains with arbitrary boundaries.

Apply the Radó - Markushevich theorem to $g_{n} \circ p$ with a conformal mapping $p$ from $\mathbb{D}$ onto $\mathbb{H}$ and obtain that the sequence $\left\{g_{n}(w, t)\right\}$ converges to $f_{c}^{-1}(w, t)$ as $n \rightarrow \infty$ uniformly on compact subsets of $\mathbb{H} \cup \mathbb{R}$.

Denote by $\Gamma_{1}[0, \tau(t)]$ the left side of the segment $I_{\theta(c)}(|B(c)| \sqrt{\tau})$ and denote by $\Gamma_{2}[0, \tau(t)]$ the right side of this segment. Similarly, denote $\gamma_{1 n}[0, t]$ the left side of $\gamma^{(n)}[0, t]$, and denote $\gamma_{2 n}[0, t]$ the right side of $\gamma^{(n)}[0, t]$. The functions $g_{n}^{-1}(z, t)$ map $\gamma_{1 n}[0, t]$ and $\gamma_{2 n}[0, t]$ onto segments $I_{1 n}=I_{1 n}(t) \subset \mathbb{R}$ and $I_{2 n}=I_{2 n}(t) \subset \mathbb{R}$, respectively. It is known, see, e.g. [5], that slit sides $\Gamma_{1}[0, \tau(t)]$ and $\Gamma_{2}[0, \tau(t)]$ are mapped by $f_{c}(z, t)$ onto

$$
I_{1}=I_{1}(t)=\left[\frac{c-\sqrt{c^{2}+16}}{2} \sqrt{t}, c \sqrt{t}\right] \quad \text { and } \quad I_{2}=I_{2}(t)=\left[c \sqrt{t}, \frac{c+\sqrt{c^{2}+16}}{2} \sqrt{t}\right],
$$

respectively. The uniform convergence of $g_{n}$ to $f_{c}^{-1}$ implies that $I_{1 n}(t)$ tend to $I_{1}(t)$, and $I_{2 n}(t)$ tend to $I_{2}(t)$ as $n \rightarrow \infty$.

Denote by $\gamma_{1 n}^{\prime}[0, t]$ and $\gamma_{2 n}^{\prime}[0, t]$ the left and the right sides of $\gamma\left[0, \frac{t}{x_{n}}\right]$, respectively. The function $f\left(z, \frac{t}{x_{n}}\right)$ maps slit sides $\gamma_{1 n}^{\prime}[0, t]$ and $\gamma_{2 n}^{\prime}[0, t]$ onto segments $I_{1 n}^{\prime}=I_{1 n}^{\prime}(t) \subset \mathbb{R}$ and $I_{2 n}^{\prime}=I_{2 n}^{\prime}(t) \subset \mathbb{R}$, respectively. Compare $I_{k n}(t)$ and $I_{k n}^{\prime}(t)$ by $(7)$ and conclude that $I_{k n}(t)=\sqrt{x_{n}} I_{k n}^{\prime}(t)$, and so

$$
\text { meas } I_{k n}(t)=\sqrt{x_{n}} \text { meas } I_{k n}^{\prime}(t), \quad k=1,2, \quad n \geq 1, \quad 0<t \leq T .
$$

The harmonic measure is invariant under conformal transformations. This gives that

$$
\frac{m_{1}\left(\frac{t}{x_{n}}\right)}{m_{2}\left(\frac{t}{x_{n}}\right)}=\frac{\omega\left(f^{-1}\left(i, \frac{t}{x_{n}}\right), \gamma_{1}\left[0, \frac{t}{x_{n}}\right], \mathbb{H} \backslash \gamma\left(\frac{t}{x_{n}}\right)\right)}{\omega\left(f^{-1}\left(i, \frac{t}{x_{n}}\right), \gamma_{2}\left[0, \frac{t}{x_{n}}\right], \mathbb{H} \backslash \gamma\left(\frac{t}{x_{n}}\right)\right)}=\frac{\omega\left(i, I_{1 n}^{\prime}(t), \mathbb{H}\right)}{\omega\left(i, I_{2 n}^{\prime}(t), \mathbb{H}\right)}
$$

For $k=1,2, n \geq 1$, the harmonic measure $\omega\left(i ; I_{k n}^{\prime}(t), \mathbb{H}\right)$ of $I_{k n}^{\prime}(t)$ at $i$ with respect to $\mathbb{H}$ equals the angle divided over $\pi$ under which the segment $I_{k n}^{\prime}(t)$ is seen from the point $i$. Similarly, the harmonic measure $\omega\left(i ; I_{k n}(t), \mathbb{H}\right)$ of $I_{k n}(t)$ at $i$ with respect to $\mathbb{H}$ equals the angle divided over $\pi$ under which the segment $I_{k n}(t)$ is seen from the point $i$, see, e.g. [8, p. 334]. This shows that the last term in the chain of equalities has a limit as $n \rightarrow \infty$, and

$$
\lim _{n \rightarrow \infty} \frac{\omega\left(i, I_{1 n}^{\prime}(t), \mathbb{H}\right)}{\omega\left(i, I_{2 n}^{\prime}(t), \mathbb{H}\right)}=\lim _{n \rightarrow \infty} \frac{\tan \left(\pi \omega\left(i, I_{1 n}^{\prime}(t), \mathbb{H}\right)\right)}{\tan \left(\pi \omega\left(i, I_{2 n}^{\prime}(t), \mathbb{H}\right)\right)}=\lim _{n \rightarrow \infty} \frac{\operatorname{meas} I_{1 n}^{\prime}(t)}{\operatorname{meas} I_{2 n}^{\prime}(t)}=\lim _{n \rightarrow \infty} \frac{\operatorname{meas} I_{1 n}(t)}{\operatorname{meas} I_{2 n}(t)}
$$

This limit exists for every sequence $\left\{x_{n}\right\}$ tending to infinity. So there exists a limit for the ratio of $m_{1}(t)$ and $m_{2}(t)$ as $t \rightarrow+0$, and

$$
\lim _{t \rightarrow+0} \frac{m_{1}(t)}{m_{2}(t)}=\lim _{n \rightarrow \infty} \frac{m_{1}\left(\frac{t}{n}\right)}{m_{2}\left(\frac{t}{n}\right)}=\lim _{t \rightarrow+0} \frac{\operatorname{meas} I_{1}(t)}{\operatorname{meas} I_{2}(t)}=\frac{\sqrt{c^{2}+16}+c}{\sqrt{c^{2}+16}-c}=\frac{1+\beta}{1-\beta},
$$

where $\beta$ is given by (3). This leads to the conclusion desired in Theorem 1 and completes the proof.

\section{PROOF OF THEOREM 2}

Proof of Theorem 2. The Löwner equation (1) admits an explicit integration [10] in the case when $\gamma_{0}[0, t]$ is a circular arc centered at $i, \gamma_{0}(0)=0$, with an implicitly given driving function $\lambda(t)$. To be concrete, we will consider $\gamma_{0}[0, t]$ such that the argument of $\left(\gamma_{0}[0, t]-i\right)$ increases in $t$. Let a 
solution $f_{0}(z, t)$ to equation (1) map $\mathbb{H} \backslash \gamma_{0}[0, t]$ onto $\mathbb{H}$. Its inverse $f_{0}^{-1}(w, t)$ is represented [10] by the Christoffel - Schwarz integral

$$
\frac{1}{f_{0}^{-1}(w, t)}=\int_{0}^{\frac{1}{w}} \frac{\left(1-\lambda_{0} w\right) d w}{\left(1-\beta_{1} w\right)^{2}\left(1-\beta_{2} w\right)}=\frac{1}{2 \pi} \log \frac{w-\beta_{1}}{w-\beta_{2}}+\frac{\beta_{2}+\beta_{1}}{\beta_{2}-\beta_{1}} \frac{1}{w-\beta_{1}},
$$

where $\beta_{1}=\beta_{1}(t)$ and $\beta_{2}=\beta_{2}(t)$ are expanded in powers of $\sqrt[3]{t}$,

$$
\beta_{1}(t)=A_{1} \sqrt[3]{t^{2}}+A_{2} t+A_{3} \sqrt[3]{t^{4}}+\ldots, \quad A_{1}=-\frac{\sqrt[3]{9}}{\sqrt[3]{4 \pi}},
$$

and

$$
\beta_{2}(t)=B_{1} \sqrt[3]{t}+B_{2} \sqrt[3]{t^{2}}+\ldots, \quad B_{1}=\sqrt[3]{12 \pi} .
$$

The driving function $\lambda_{0}(t)$ is evaluated by

$$
\lambda_{0}(t)=2 \beta_{1}(t)+\beta_{2}(t)=C_{1} \sqrt[3]{t}+C_{2} \sqrt[3]{t^{2}}+\ldots, \quad C_{1}=B_{1} .
$$

Suppose that a $C^{6}$-slit $\gamma_{t}$ satisfies the conditions of Theorem 2. Then there exists a driving function $\lambda(t)$ such that a solution $w=f(z, t)$ to equation (1) maps $\mathbb{H} \backslash \gamma_{t}$ onto $\mathbb{H}$. For the arc-length parameter $s$, represent a transformation of $\gamma(t(s))$,

$$
\tilde{\gamma}(s):=\frac{2 \gamma(t(s))}{2+i \gamma(t(s))}=s+o\left(s^{6}\right), \quad s \rightarrow+0 .
$$

The function $f_{0}(z, \tau)$ maps $\mathbb{H} \backslash \gamma_{0}[0, \tau]$ onto $\mathbb{H}$. Hence,

$$
G_{0}(w, \tau)=\frac{2 f_{0}^{-1}(w, \tau)}{2+i f_{0}^{-1}(w, \tau)}
$$

maps $\mathbb{H}$ onto the exterior of the disk of radius 1 centered at $(-i)$ and slit along the segment $[0, \sigma] \subset \mathbb{R}$. The length $\sigma(\tau)$ of $[0, \sigma]$ and the half-plane capacity $\tau$ of $\gamma_{0}[0, \tau]$ are related by

$$
\sigma(\tau)=\frac{2 f_{0}^{-1}\left(\lambda_{0}(\tau), \tau\right)}{2+i f_{0}^{-1}\left(\lambda_{0}(\tau), \tau\right)}=B_{1} \sqrt[3]{\tau}+o\left(\sqrt[3]{\tau^{2}}\right), \quad \tau \rightarrow+0 .
$$

Let $s$ denote the length of $\tilde{\gamma}[0, s]$, and let $\sigma$ denote the length of projection of $\tilde{\gamma}[0, s]$ onto $[0, \sigma]$. There is a $C^{6}$-dependence $s=s(\sigma)$,

$$
s(0)=0, \quad s^{\prime}(0)=1, \quad s^{(k)}(0)=0, k=2, \ldots, 6 .
$$

Therefore,

$$
s=\sigma+o\left(\sigma^{6}\right), \quad \sigma \rightarrow+0 .
$$

Asymptotic expansion (8) implies an asymptotic behavior of a distance between $\tilde{\gamma}$ and its projection on $[0, \sigma]$,

$$
\operatorname{dist}(\tilde{\gamma}[0, s],[0, \sigma(s)])=o\left(\sigma^{6}(s)\right), \quad s \rightarrow+0 .
$$

According to Lemma 4.10 [2], we have that

$$
t(s)-\tau(\sigma(s))=o\left(s^{3}\right), \quad s \rightarrow+0,
$$

where $\sigma(\tau)$ is given by (9). Hence, due to (9) and (10),

$$
t(s(\sigma))=\tau(\sigma)+o\left(s^{3}(\sigma)\right)=\tau(\sigma)+o\left(\sigma^{3}\right)=B_{1}^{-3} s^{3}+o\left(s^{3}\right), \quad s \rightarrow+0 .
$$

Take into account (8) and rewrite the last relation in the form

$$
\gamma(t)=B_{1} \sqrt[3]{t}+\alpha(t) \sqrt[3]{t}, \quad \lim _{t \rightarrow+0} \alpha(t)=0 .
$$


Choose an arbitrary sequence $\left\{x_{n}\right\}$ of positive numbers $x_{n}, x_{n} \rightarrow \infty$ as $n \rightarrow \infty$, and denote

$$
z=g_{n}(w, t):=\sqrt{x_{n}} f^{-1}\left(\frac{w}{\sqrt{x_{n}}}, \frac{t}{x_{n}}\right), \quad n=1,2, \ldots .
$$

The function $z=f^{-1}(w, t)$ maps $\mathbb{H}$ onto $\mathbb{H} \backslash \gamma[0, t], f^{-1}(\lambda(t), t)=\gamma(t)$. So the functions

$$
G_{n}(w, t):=\frac{2 g_{n}(w, t)}{2+i g_{n}(w, t)}, \quad n=1,2, \ldots,
$$

map $\mathbb{H}$ onto the exterior of the disk of radius 1 centered at $(-i)$ minus $\tilde{\gamma}^{(n)}(t)$ where

$$
\begin{gathered}
\tilde{\gamma}^{(n)}(t)=\sqrt{x_{n}} \gamma\left(\frac{t}{x_{n}}\right)=B_{1} \sqrt[3]{t}+\alpha\left(\frac{t}{x_{n}}\right) \sqrt[3]{t}, \\
G_{n}\left(\sqrt{x_{n}} \lambda\left(\frac{t}{x_{n}}\right), t\right)=\tilde{\gamma}^{(n)}(t), \quad 0<t \leq T .
\end{gathered}
$$

We see that

$$
\tilde{\gamma}^{(n)}(t)-B_{1} \sqrt[3]{t}=\alpha\left(\frac{t}{x_{n}}\right) \sqrt[3]{t} \rightarrow 0, \quad n \rightarrow \infty,
$$

and the convergence is uniform with respect to $t \in[0, T]$.

Apply the Radó - Markushevich theorem to $g_{n} \circ p$ with a conformal mapping $p$ from $\mathbb{D}$ onto $\mathbb{H}$ and obtain that the sequence $\left\{G_{n}(w, t)\right\}$ converges to $G_{0}(w, t)$ which implies that $\left\{g_{n}(w, t)\right\}$ converges to $f_{0}^{-1}(w, t)$ as $n \rightarrow \infty$ uniformly on compact subsets of $\mathbb{H} \cup \mathbb{R}$.

Denote by $\Gamma_{1}[0, \tau(t)]$ the left side of the circular arc $\gamma_{0}[0, \tau]$ and denote by $\Gamma_{2}[0, \tau(t)]$ the right side of this circular arc. Similarly, denote $\gamma_{1 n}[0, t]$ the left side of

$$
\gamma^{(n)}[0, t]:=\frac{2 \tilde{\gamma}[0, t]}{2-i \tilde{\gamma}[0, t]}
$$

and denote $\gamma_{2 n}[0, t]$ the right side of $\gamma^{(n)}[0, t]$. The functions $g_{n}^{-1}(z, t)$ map $\gamma_{1 n}[0, t]$ and $\gamma_{2 n}[0, t]$ onto segments $I_{1 n}=I_{1 n}(t) \subset \mathbb{R}$ and $I_{2 n}=I_{2 n}(t) \subset \mathbb{R}$, respectively. It is shown [10] that slit sides $\Gamma_{1}[0, \tau(t)]$ and $\Gamma_{2}[0, \tau(t)]$ are mapped by $f_{0}(z, t)$ onto

$$
I_{1}=I_{1}(t)=\left[\beta_{1}(t), \lambda_{0}(t)\right] \quad \text { and } \quad I_{2}=I_{2}(t)=\left[\lambda_{0}(t), \beta_{2}(t)\right],
$$

respectively. The uniform convergence of $g_{n}$ to $f_{0}^{-1}$ implies that $I_{1 n}(t)$ tend to $I_{1}(t)$, and $I_{2 n}(t)$ tend to $I_{2}(t)$ as $n \rightarrow \infty$.

Denote by $\gamma_{1 n}^{\prime}[0, t]$ and $\gamma_{2 n}^{\prime}[0, t]$ the left and the right sides of $\gamma\left[0, \frac{t}{x_{n}}\right]$, respectively. The function $f\left(z, \frac{t}{x_{n}}\right)$ maps slit sides $\gamma_{1 n}^{\prime}[0, t]$ and $\gamma_{2 n}^{\prime}[0, t]$ onto segments $I_{1 n}^{\prime}=I_{1 n}^{\prime}(t) \subset \mathbb{R}$ and $I_{2 n}^{\prime}=I_{2 n}^{\prime}(t) \subset \mathbb{R}$, respectively. Compare $I_{k n}(t)$ and $I_{k n}^{\prime}(t)$ by $(12)$ and conclude that $I_{k n}(t)=\sqrt{x_{n}} I_{k n}^{\prime}(t)$, and so

$$
\text { meas } I_{k n}(t)=\sqrt{x_{n}} \text { meas } I_{k n}^{\prime}(t), \quad k=1,2, \quad n \geq 1, \quad 0<t \leq T .
$$

The harmonic measure is invariant under conformal transformations. This gives that

$$
\frac{M_{1}^{2}\left(\frac{t}{x_{n}}\right)}{M_{2}\left(\frac{t}{x_{n}}\right)}=\frac{\omega^{2}\left(f^{-1}\left(i, \frac{t}{x_{n}}\right), \gamma_{1}\left[0, \frac{t}{x_{n}}\right], \mathbb{H} \backslash \gamma\left(\frac{t}{x_{n}}\right)\right)}{\omega\left(f^{-1}\left(i, \frac{t}{x_{n}}\right), \gamma_{2}\left[0, \frac{t}{x_{n}}\right], \mathbb{H} \backslash \gamma\left(\frac{t}{x_{n}}\right)\right)}=\frac{\omega^{2}\left(i, I_{1 n}^{\prime}(t), \mathbb{H}\right)}{\omega\left(i, I_{2 n}^{\prime}(t), \mathbb{H}\right)}
$$

For $k=1,2, n \geq 1$, the harmonic measure $\omega\left(i ; I_{k n}^{\prime}(t), \mathbb{H}\right)$ of $I_{k n}^{\prime}(t)$ at $i$ with respect to $\mathbb{H}$ equals the angle divided over $\pi$ under which the segment $I_{k n}^{\prime}(t)$ is seen from the point $i$. Similarly, the harmonic measure $\omega\left(i ; I_{k n}(t), \mathbb{H}\right)$ of $I_{k n}(t)$ at $i$ with respect to $\mathbb{H}$ equals the angle divided over $\pi$ under which the segment $I_{k n}(t)$ is seen from the point $i$. This shows that the last term in the chain of equalities has a limit as $n \rightarrow \infty$ and

$$
\lim _{n \rightarrow \infty} \frac{\omega^{2}\left(i, I_{1 n}^{\prime}(t), \mathbb{H}\right)}{\omega\left(i, I_{2 n}^{\prime}(t), \mathbb{H}\right)}=\lim _{n \rightarrow \infty} \frac{\tan ^{2}\left(\pi \omega\left(i, I_{1 n}^{\prime}(t), \mathbb{H}\right)\right)}{\tan \left(\pi \omega\left(i, I_{2 n}^{\prime}(t), \mathbb{H}\right)\right)}=\lim _{n \rightarrow \infty} \frac{\operatorname{meas}^{2} I_{1 n}^{\prime}(t)}{\operatorname{meas} I_{2 n}^{\prime}(t)}=\lim _{n \rightarrow \infty} \frac{\operatorname{meas}^{2} I_{1 n}(t)}{\operatorname{meas} I_{2 n}(t)}
$$


This limit exists for every sequence $\left\{x_{n}\right\}$ tending to infinity. So there exists a limit for the ratio of $M_{1}^{2}(t)$ and $M_{2}(t)$ as $t \rightarrow+0$, and

$$
\begin{gathered}
\lim _{t \rightarrow+0} \frac{M_{1}^{2}(t)}{M_{2}(t)}=\lim _{n \rightarrow \infty} \frac{M_{1}^{2}\left(\frac{t}{n}\right)}{M_{2}\left(\frac{t}{n}\right)}=\lim _{t \rightarrow+0} \frac{\operatorname{meas}^{2} I_{1}(t)}{\operatorname{meas} I_{2}(t)}=\lim _{t \rightarrow+0} \frac{\left(\lambda_{0}(t)-\beta_{1}(t)\right)^{2}}{\beta_{2}(t)-\lambda_{0}(t)}= \\
=\lim _{t \rightarrow+0} \frac{\left(B_{1} \sqrt[3]{t}+\left(C_{2}-A_{1}\right) \sqrt[3]{t^{2}}+\ldots\right)^{2}}{\left(B_{2}-C_{2}\right) \sqrt[3]{t^{2}}+\ldots}=\frac{B_{1}^{2}}{B_{2}-C_{2}}=\frac{B_{1}^{2}}{-2 A_{1}}=2 \pi .
\end{gathered}
$$

This leads to the conclusion desired in Theorem 2 and completes the proof.

D. V. Prokhorov has been supported by the RF Ministry of Education and Science (project no. 1.1520.2014K). D. V. Ukrainskii has been supported by the Russian/Turkish grant RFBR/TUBITAk no. 14-01-91370.

\title{
References
}

1. Löwner K. Untersuchungen über schlichte konforme Abbildungen des Einheitskreises. I. Math. Ann., 1923, vol. 89, no. 1-2, pp. 103-121.

2. Lind J., Marshall D. E., Rohde S. Collisions and spirals of Loewner traces. Duke Math. J., 2010, vol. 154 , no. 3 , pp. 527-573.

3. Hayman W. K., Kennedy P. B. Subharmonic Functions, vol. 1, London, New York, Academic Press, 1976.

4. Earle C. J., Epstein A. L. Quasiconformal variation of slit domains. Proc. Amer. Math. Soc., 2001, vol. 129, no. 11, pp. 3363-3372.

5. Prokhorov D., Zakharov A. Harmonic measures of sides of a slit perpendicular to the domain boundary. J. Math. Anal. Appl., 2012, vol. 394, no. 2 , pp. $738-743$.

6. Kager W., Nienhuis B., Kadanoff L. P. Exact solutions for Loewner evolutions, J. Statist. Phys., 2004, vol. 115 , no. 3-4, pp. 805-822.
7. Radó T. Sur la représentations conforme de domaines variables. Acta Sci. Math. (Szeged), 1922-1923, vol. 1, no. 3, pp. 180-186.

8. Goluzin G. M. Geometric Theory of Functions of Complex Variables. Moscow, Nauka, 1966.

9. Markushevich A. I. Sur la représentations conforme des domaines à frontières variables. Rec. Math. [Mat. Sbornik] N.S., 1936, vol. 1(43), no. 6, pp. 863-886.

10. Prokhorov D., Vasil'ev A. Singular and tangent slit solutions to the Löwner equation. Analysis and Mathematical Physics, eds. D. Gustafsson, A. Vasil'ev. Berlin, Birkhauser, 2009, pp. 455-463.

11. Ivanov G., Prokhorov D., Vasil'ev A. Non-slit and singular solutions to the Löwner equation. Bull. Sci. Mathem., 2012, vol. 136, no. 3, pp. 328-341.

УДК 517.54

\section{Асимптотическое отношение гармонических мер сторон разреза}

\section{Д. В. Прохоров ${ }^{1}$, Д. В. Украинский ${ }^{2}$}

\footnotetext{
${ }^{1}$ Доктор фризико-математических наук, заведующий кафредрой математического анализа, Саратовский государственный университет им. Н. Г. Чернышевского, ProkhorovDV @info.sgu.ru

${ }^{2}$ Студент механико-математического фракультета, Саратовский государственный университет им. Н. Г. Чернышевского, D.V.Ukrainskiy@gmail.com
}

\begin{abstract}
Статья посвящена геометрии решений хордового уравнения Левнера, основанной на сравнении сингулярных решений и гармонических мер берегов разреза в верхней полуплоскости, порожденного управляющей фрункцией. Найдено асимптотическое отношение гармонических мер берегов разреза, касательного к прямой под заданным углом, и разреза, имеющего высокий порядок касания к дуге окружности, касающейся действительной оси.
\end{abstract}

Ключевые слова: уравнение Левнера, сингулярное решение, гармоническая мера, емкость в полуплоскости.

Д. В. Прохоров получил поддержку Министерства образования и науки РФ (проект № 1.1520.2014K). Д. В. Украинский поддержан российско-турецкким грантом РФФИ/ТЮБИТАК № 14-01-91370. 


\title{
Библиографичческий список
}

1. Löwner $K$. Untersuchungen über schlichte konforme Abbildungen des Einheitskreises. I // Math. Ann. 1923. Vol. 89, № 1-2. P. 103-121.

2. Lind J., Marshall D. E., Rohde S. Collisions and spirals of Loewner traces // Duke Math. J. 2010. Vol. 154, № 3. P. 527-573.

3. Hayman $W$. K., Kennedy P. B. Subharmonic Functions. Vol. 1. London, New York : Academic Press, 1976.

4. Earle C. J., Epstein A. L. Quasiconformal variation of slit domains // Proc. Amer. Math. Soc. 2001. Vol. 129, № 11. P. 3363-3372.

5. Prokhorov D., Zakharov A. Harmonic measures of sides of a slit perpendicular to the domain boundary // J. Math. Anal. Appl. 2012. Vol. 394, № 2. P. $738-743$.

6. Kager W., Nienhuis B., Kadanoff L. P. Exact solutions for Loewner evolutions // J. Statist. Phys. 2004. Vol. 115, № 3-4. P. 805-822.
7. Radó T. Sur la représentations conforme de domaines variables // Acta Sci. Math. (Szeged). 1922-1923. Vol. 1, № 3. P. 180-186.

8. Голузин Г. М. Геометрическая теория функций комплексного переменного. М. : Наука, 1966.

9. Markouchevitch $A$. Sur la représentation conforme des domaines à frontières variables // Матем. сб. 1936. T. 1(43), № 6. C. 863-886.

10. Prokhorov D., Vasil'ev A. Singular and tangent slit solutions to the Löwner equation // Analysis and Mathematical Physics / eds. D. Gustafsson, A. Vasil'ev. Berlin : Birkhauser, 2009. P. 455-463.

11. Ivanov G., Prokhorov D., Vasil'ev A. Non-slit and singular solutions to the Löwner equation // Bull. Sci. Mathem. 2012. Vol. 136, № 3. P. 328-341.

УДК 517.584

\section{О НЕКОТОРЫХ ИНТЕГРАЛЬНЫХ СВОЙСТВАХ МОДИФИЦИРОВАННЫХ ФУНКЦИЙ БЕССЕЛЯ}

\section{Ю. М. Раппопорт}

\begin{abstract}
Кандидат фризико-математических наук, доцент, старший научный сотрудник, Институт автоматизации проектирования Российской Академии наук, Москва, jmrap @landau.ac.ru

Представлены новые интегральные тождества для модифицированных функций Бесселя произвольного комплексного порядка. Изучены свойства интегральных преобразований Лебедева - Скальской.
\end{abstract}

Ключевые слова: модисрицированные фрункции Бесселя комплексного порядка, интегральные преобразования Конторовича - Лебедева, интегральные преобразования Лебедева - Скальской.

\section{1. НЕКОТОРЫЕ СВОЙСТВА ФУНКЦИЙ $\operatorname{Re} K_{\alpha+i \beta}(x)$ И $\operatorname{Im} K_{\alpha+i \beta}(x)$}

Можем записать вещественную и мнимую часть модифицированных функций Бесселя комплексного порядка в виде

$$
\operatorname{Re} K_{\alpha+i \beta}(x)=\frac{K_{\alpha+i \beta}(x)+K_{\alpha-i \beta}(x)}{2} \quad \text { и } \quad \operatorname{Im} K_{\alpha+i \beta}(x)=\frac{K_{\alpha+i \beta}(x)-K_{\alpha-i \beta}(x)}{2 i},
$$

где $K_{\nu}(x)$ - модифицированная функция Бесселя второго рода (также называемая функцией Макдональда).

Функции $K_{i \beta}(x), \operatorname{Re} K_{\alpha+i \beta}(x)$ и $\operatorname{Im} K_{\alpha+i \beta}(x)$ имеют интегральные представления $[1,2]$

$$
\begin{aligned}
K_{i \beta}(x) & =\int_{0}^{\infty} e^{-x \cosh t} \cos (\beta t) d t \\
\operatorname{Re} K_{\alpha+i \beta}(x) & =\int_{0}^{\infty} e^{-x \cosh t} \cosh (\alpha t) \cos (\beta t) d t \\
\operatorname{Im} K_{\alpha+i \beta}(x) & =\int_{0}^{\infty} e^{-x \cosh t} \sinh (\alpha t) \sin (\beta t) d t .
\end{aligned}
$$

Из (1), (2) следует, что возможно переписать $\operatorname{Re} K_{\alpha+i \beta}(x)$ в виде косинус-преобразования Фурье

$$
\operatorname{Re} K_{\alpha+i \beta}(x)=\left(\frac{\pi}{2}\right)^{1 / 2} F_{C}\left[e^{-x \cosh t} \cosh (\alpha t) ; t \rightarrow \beta\right]
$$

